\title{
Monocyte/High Density Lipoprotein Ratio in Patients with Symptomatic Carotid Artery Stenosis and Its Relationship with Stenosis Degree
}

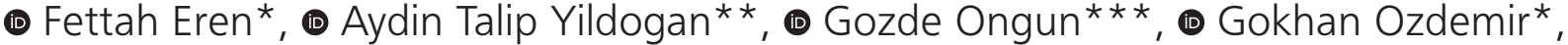 \\ (1) Serefnur Ozturk* \\ *Selcuk University Faculty of Medicine, Department of Neurology, Konya, Turkey \\ **Ersin Arslan Training and Research Hospital, Clinic of Neurology, Gaziantep, Turkey \\ $* *$ Mus State Hospital, Clinic of Neurology, Mus, Turkey
}

\section{Abstract}

\begin{abstract}
Aim: Monocyte/high-density lipoprotein (HDL) ratio (MHR) is an inflammatory marker associated with vascular diseases. The aim of this study is to evaluate the MHR in patients with symptomatic carotid artery stenosis and to examine its relationship with the degree of stenosis.

Methods: The study was conducted as a case-control study, with data obtained from patients' files between January 2020 and May 2021. Ninety patients with symptomatic carotid stenosis and 40 healthy controls were included in the study. For vascular imaging, computed tomography angiography and/or digital subtraction angiography were used. Carotid artery stenosis was grouped according to North American Symptomatic Carotid Endarterectomy Trial criteria. Tests for monocyte and HDL cholesterol were performed, and the MHR was calcu-lated.
\end{abstract}

Results: There were 67 male and 23 female patients with a mean age of $65.10 \pm 12.26$ years in the study. In 46 (51.1\%) of the patients, there was $50 \%$ or more carotid artery stenosis. The HDL cholesterol level was lower, and the MHR was higher in the patient group ( $p=0.042, p=0.041$, respectively). Monocyte and MHR levels were higher in patients with $50 \%$ or more carotid stenosis ( $p=0.04)$.

Conclusion: The MHR is a predictive biomarker for the degree of symptomatic stenosis in the carotid artery.

Keywords: Ischemic attack, carotid stenosis, monocyte/HDL ratio, monocytes, cholesterol

\section{Introduction}

An acute ischemic stroke describes a focal or global cerebral injury that develops suddenly and has no apparent cause other than vascular. Ischemia is the most common etiological cause of strokes, and carotid artery disease is the cause in approximately one-third of them. It occurs especially with atherosclerosis (1-3). There is evidence that the inflammatory process associated with atherosclerosis and vascular stenosis is important in the development and prognosis of ischemic stroke (4).

Macrophages and monocytes have critical roles in the release of proinflammatory cytokines and the modulation of inflammation $(5,6)$. Activated monocytes play a role in the etiology of cardiovascular diseases by modulating inflammatory cytokines (7). High-density lipoprotein $(\mathrm{HDL})$ tries to prevent the occurrence of endothelial damage. It does this by inhibiting the oxidation of lowdensity lipoprotein (LDL). HDL cholesterol exerts its antiinflammatory effect in this way (8). The monocyte/HDL cholesterol ratio (MHR) is an indicator of atherogenic and antiatherogenic balance, and its higher level is associated with the degree of atherosclerosis. Recent studies show that MHR may be a new predictive factor in predicting cardiovascular disease prognosis $(9,10)$. There are quite a few studies in the literature evaluating the role of MHR in the process or prognosis of ischemic stroke, but there is no study to evaluate the relationship between symptomatic carotid stenosis and MHR (11). 
This study aims to evaluate MHR in patients with symptomatic carotid artery stenosis and to reveal its relationship with the degree of stenosis.

\section{Methods}

\section{Ethical Approval and Field of Study}

Ischemic stroke patients hospitalized in the neurology clinic were included in the study, which was planned as a case-control and retrospective study. The data were obtained with patients file from January 2020 to May 2021. Since this study was retrospective, an informed consent form was not required. Prior to the study, approval was obtained from the local ethics committee of Selcuk University (date: 21.04.2021, decision number: 2021/221). The study process adhered to the Helsinki Declaration and good clinical practice guidelines.

\section{Participants}

A total of 800 ischemic stroke patients were evaluated. Patients with temporary or permanent carotid region stroke symptoms within the last 6 months were evaluated. Ninety stroke patients and 40 control patients who were found to have stenosis in the carotid location consistent with the present symptom on vascular imaging were included in the study. Age, gender and chronic diseases [hypertension, diabetes mellitus (DM)] were evaluated. Patients were grouped as under 65 years old, 66-79 years old and over 80 years old. The control patients included in the study were healthy individuals of similar age and gender as the patient group. In addition, these patients did not have the exclusion criteria and stroke history. The study design and exclusion criteria were determined (Figure 1). It was thought that these factors might change monocytes and HDL cholesterol levels. They were pregnancy, active systemic infection, hematological disease and antihyperlipidemic treatment history. Patients with other stroke risk factors were excluded. They were vertebrobasilar and intracranial stenosis, mechanical prosthetic valve, severe mitral stenosis, atrial fibrillation, intracardiac thrombus, endocarditis, dilated cardiomyopathy and atrial myxoma.

\section{Brain Computed Tomography Angiography}

CTA was used to determine the degree and location of carotid artery stenosis and was performed with 64

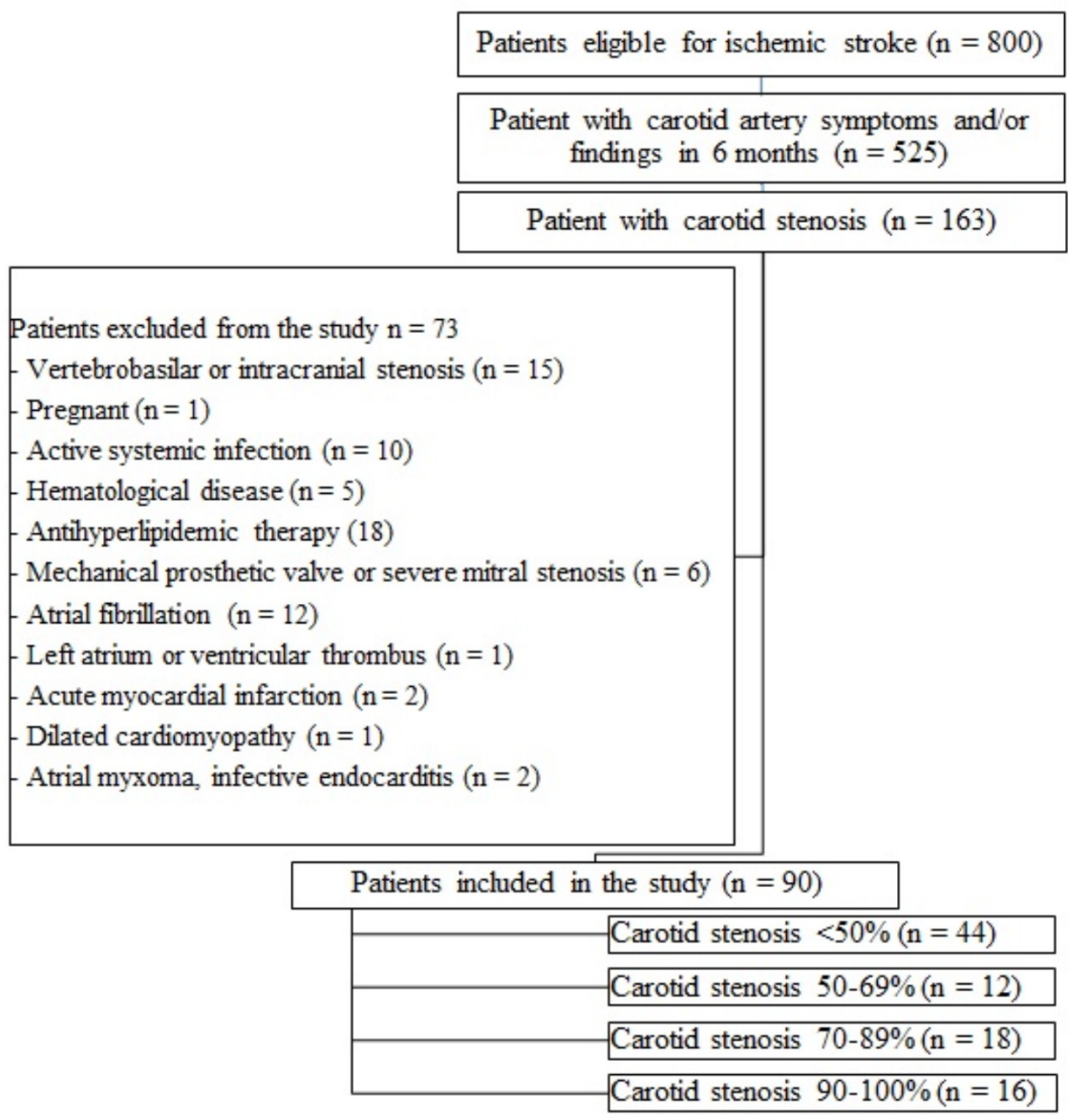

Figure 1. Study design and exclusion criteria 
MDCT (Toshiba, Japan). The arcus aorta and the sphenoid wing are scanned in the upper section. The scanning area is determined, and the recording is made by giving contrast material via the venous route. Various programs and reconstructions were obtained by volume rendering techniques. Calcifications and stenosis degrees are determined with various window settings.

\section{Digital Subtraction Angiography}

Digital subtraction angiography (DSA) was used in addition to computed tomography angiography in patients whose stenosis degree and localization in the carotid artery could not be determined decisively. The Axiom Artis FA (Siemens, Germany) brand device was used for DSA and the femoral artery was utilized for intervention. Sheaths were placed in sizes varying between 6F-8F. Aortography was performed first with a $6 \mathrm{~F}$ pigtail from the aortic arch. In cases where both carotid arteries (AP and lateral) were required, images were taken obliquely. Non-ionic contrast material was used to obtain the images.

\section{Evaluation of Images}

The stenosis degrees of the patients included in the study were determined by tomography angiography and/or DSA. The degree of carotid artery stenosis was calculated according to the North American Symptomatic Carotid Endarterectomy Trial (NASCET) criteria. The degree of stenosis was calculated using the formula: (1-narrowest segment/distal normal segment) x $100(12,13)$. According to the NASCET, the results were divided into 4 groups: less than 50\%, 50-69, 70-89, and 90 and above. The localization of carotid artery stenosis was determined (right and/or left).

\section{Blood Test Parameters}

Monocyte and HDL levels were detected at any time point within six months after stroke symptoms. The MHR was calculated with these levels. Blood samples were taken on hospitalization, in the morning hours after fasting for at least 8 hours. Serum was taken into gel tubes with a separator and blood samples were taken into tubes with potassium-ethylenediaminetetraacetic acid. The samples obtained (5000 rpm) were centrifuged and sera were separated. HDL cholesterol spectrophotometry, the parameter used in the study, was measured using hemogram (monocyte) fluorescence flow cytometry and was calculated using the formula MHR=Monocyte $(\mathrm{K} / \mathrm{UL}) /$ $\mathrm{HDL}$ cholesterol (mg/dL) x 1000 .

\section{Statistical Analysis}

SPSS 16.0 Package Software (Statistical Package for the Social Sciences Inc.; Armonk, NY, USA) was used to analyze the collected data. Normality analysis was performed using the Kolmogorov-Smirnov test. The demographic data obtained were expressed as a number, percentage, mean standard deviation, or median (minimum-maximum) based on the results of the normality analysis. Normally distributed data was compared with the Student's t-test. Data that was not normally distributed was analyzed using the Kruskal-Wallis test. Post-hoc analysis was performed using the Mann-Whitney $U$ test and Bonferroni correction. The chi-square, or Fisher's exact test, was used to compare categorical data. The MHR cut-off points were calculated according to the receiver operating characteristic (ROC) curve. The cut-off point was determined according to the sensitivity and specificity values. The area under the curve (AUC) was calculated with confidence intervals $(\mathrm{Cl})$. The relationship between the numerical data was evaluated using Spearman's correlation test. A logistic regression analysis was used to predict the factors affecting carotid artery stenosis. The results were considered significant at $\mathrm{p}<0.05$ with a $95 \% \mathrm{Cl}$.

\section{Results}

In the study, there were 90 carotid artery stenosis patients with a mean age of $65.10 \pm 12.26$ (38-86) years and 40 healthy volunteers with a mean age of $64.03 \pm 9.481$ (50-78) years. Twenty-three $(25.6 \%)$ of the patients with carotid stenosis were female and 67 $(74.4 \%)$ were male. Patients and healthy controls were comparable in terms of age and gender $(p=0.098)$. The patients had lower HDL cholesterol levels $(p=0.042)$ and higher MHR levels $(p=0.041)$ compared with the control group. Clinical and laboratory parameters in patients with symptomatic carotid artery stenosis and the control group are summarized in (Table 1).

There was no difference in monocyte, HDL cholesterol, and MHR levels between the age groups (under 65 years old, 66-79 years old and over 80 years old) $(p=0.33$, $p=0.60$, and $p=0.39$, respectively). When the patients were grouped according to their gender, it was found that there was no difference in the levels of monocyte, HDL cholesterol and MHR ( $p=0.05, p=0.39$, and $p=0.06$, respectively). No relationship was found between the presence of hypertension or DM and the level of monocytes, HDL cholesterol, and MHR. There was no correlation between stenosis localization (right or left) and serum MHR level. When the degree of stenosis was examined by dividing into 4 groups (less than 50\%, 50$69 \%, 70-89 \%, 90 \%$ and above), no difference was found between the groups in terms of monocyte, HDL cholesterol and MHR levels. However, when the groups were divided into 2 groups (50\% and more, 50\% below), the monocyte and MHR levels were significantly higher in patients with $50 \%$ and more stenosis ( $p=0.04$ ) (Table 2 ). The monocyte cut-off level was calculated as 0.5285 with $68 \%$ sensitivity 
Eren et al. Monocyte/HDL Ratio in Carotid Stenosis

Table 1. Clinical and laboratory parameters in patients with symptomatic carotid artery stenosis and control group

\begin{tabular}{|c|c|c|c|}
\hline & Patient group $(n=90)$ & Healthy individuals $(n=40)$ & p-value \\
\hline Age (years), mean \pm SD & $65.10 \pm 12.26$ & $64.03 \pm 9.48$ & 0.065 \\
\hline $\begin{array}{l}\text { Female, } \mathbf{n}(\%) \\
\text { Male, } \mathbf{n}(\%)\end{array}$ & $\begin{array}{l}23(25.6) \\
67(74.4)\end{array}$ & $\begin{array}{l}16(40.0) \\
24(60.0)\end{array}$ & 0.098 \\
\hline Hypertension, n (\%) & $58(64.4)$ & $3(7.5)$ & $0.001^{*}$ \\
\hline Diabetes mellitus, n (\%) & $34(37.8)$ & $5(12.5)$ & $0.004^{*}$ \\
\hline Monocyte (K/uL), median (minimum - maximum) & $0.595(0.01-1.23)$ & $0.490(0.20-1.32)$ & 0.069 \\
\hline HDL (mg/dL), median (minimum - maximum) & $36.50(17.00-65.00)$ & $39.500(21.0-66.0)$ & $0.042 * *$ \\
\hline MHR, median (minimum - maximum) & $15.48(0.36-41.38)$ & $12.24(3.57-38.93)$ & $0.041^{* *}$ \\
\hline $\begin{array}{l}\text { Stenosis localization } \\
\text { Right, } \mathrm{n}(\%) \\
\text { Left, } \mathrm{n}(\%)\end{array}$ & $\begin{array}{l}26(57.8) \\
18(21.1)\end{array}$ & - & - \\
\hline $\begin{array}{l}\text { Stenosis degree, } \mathbf{n}(\%) \\
0-50 \% \\
50-69 \% \\
70-89 \% \\
90-100 \%\end{array}$ & $\begin{array}{l}44(48.9) \\
12(13.3) \\
18(20.0) \\
16(17.8)\end{array}$ & $\begin{array}{l}- \\
- \\
-\end{array}$ & $\begin{array}{l}- \\
- \\
- \\
-\end{array}$ \\
\hline
\end{tabular}

and $55 \%$ specificity for stenosis above and below 50\% ( $p=0.037,95 \% \mathrm{Cl}:$ 0.511-0.745, AUC: 0.628) (Figure 2). The MHR cut-off level was calculated as 15.22 with $66 \%$ sensitivity and $58 \%$ specificity for stenosis above and below 50\% ( $p=0.46,95 \%$ Cl: 0.503-0.741, AUC: 0.622) (Figure 3).

As a result of the Spearman correlation test, no correlation was found between the age of the patients and the monocyte, HDL cholesterol and MHR levels $(p=0.188, p=0.910, p=0.191, r=0.140, r=-0.012, r=0.139)$. There was also no correlation between monocyte, HDL cholesterol and MHR levels with increased stenosis levels in stenosis above 50\% $(p=0.119, p=0.546, p=0.186$, $r=0.233, r=-0.091, r=0.186)$.

A logistic regression model was created with hypertension, DM, monocyte, HDL cholesterol and MHR level to predict $50 \%$ or more carotid stenosis. The model achieved a good fit (Hosmer-Lemeshow=0.382, Nagelkerke $\left.R^{2}=0.128\right)$. Monocyte had an effect on carotid artery stenosis. Carotid artery stenosis (50\% or more) risk increased 2.8 times when monocytes increased by one unit $(p=0.013)$.

\section{Discussion}

The first step in the development of atherosclerosis is endothelial dysfunction. Macrophages and monocytes have an important role in the development of this step and the progression of atherosclerosis. Monocytes lead to the progression of the inflammatory reaction, transforming into macrophages and then into foam cells with oxidized lipids (10). Foam cells that turn into fatty streaks contribute to local inflammation and the secretion of proinflammatory cytokines. Local tissue factors induce intravascular coagulation $(14,15)$. The increase in monocytes and macrophages, which are the sources of foam cells and atherosclerosis, is a predictor of atherosclerosis and plaque formation (16-22). Monocytes

\begin{tabular}{|c|c|c|}
\hline & MHR-value & $p$-value \\
\hline $\begin{array}{l}\text { Age } \\
18-65(n=43) \\
66-79(n=36) \\
80-92(n=11)\end{array}$ & $\begin{array}{l}15.25(2.11-32.63) \\
16.09(4.29-39.68) \\
13.88(0.36-41.38)\end{array}$ & 0.39 \\
\hline $\begin{array}{l}\text { Male }(n=67) \\
\text { Female }(n=23)\end{array}$ & $\begin{array}{l}16.0(6.13-41.38) \\
13.95(0.36-23.46)\end{array}$ & 0.06 \\
\hline $\begin{array}{l}\text { Hypertension } \\
\text { None }(n=32) \\
\text { Yes }(n=58)\end{array}$ & $\begin{array}{l}15.51(6.13-32.63) \\
15.46(0.36-41.38)\end{array}$ & 0.95 \\
\hline $\begin{array}{l}\text { DM } \\
\text { None }(n=56) \\
\text { Yes }(n=34)\end{array}$ & $\begin{array}{l}15.26(0.36-41.38) \\
15.71(4.29-39.68)\end{array}$ & 0.84 \\
\hline $\begin{array}{l}\text { Localization } \\
\text { Right }(n=52) \\
\text { Left }(n=38)\end{array}$ & $\begin{array}{l}15.93(2.68-39.68) \\
15.21(0.36-41.38)\end{array}$ & 0.34 \\
\hline $\begin{array}{l}\text { Stenosis degree } \\
0-50 \%(n=44) \\
50-69 \%(n=12) \\
70-89 \%(n=18) \\
90-100 \%(n=16)\end{array}$ & $\begin{array}{l}14.65(0.36-32.63) \\
15.91(9.50-39.68) \\
18.77(10.67-23.46) \\
14.56(10.26-23.46)\end{array}$ & 0.07 \\
\hline $\begin{array}{l}\text { Stenosis degree } \\
0-50 \%(n=44) \\
50-100 \%(n=46)\end{array}$ & $\begin{array}{l}14.65(0.36-32.63) \\
16.03(9.50-41.38)\end{array}$ & $0.04^{*}$ \\
\hline \multicolumn{3}{|c|}{$\begin{array}{l}\text { *: Statistical significant value, *: Mann-Whitney U test } \\
\text { n: Number, \%: Percent, MHR: Monocyte/low-density lipoprotein ratio, DM } \\
\text { Diabetes mellitus }\end{array}$} \\
\hline
\end{tabular}


also have an important role in thrombus formation and the development of the ischemic area. Asymptomatic carotid artery stenosis and non-stenotic ischemic stroke have been linked to monocyte levels, degree of stenosis, stroke development, and prognosis $(11,18,23)$. Our study is also built on this etiopathogenetic process. Monocyte levels in the patient group were similar to those in the control group. The monocyte/high-density lipoprotein ratio, which is considered to be a more sensitive marker, was significantly higher in the patient group. However, it may also be related to the higher frequency of comorbid diseases in the patient group. The monocyte serum level and MHR were higher in symptomatic 50\% and more severe carotid artery stenosis. This suggests that the presence and degree of stenosis in stroke may be associated with inflammation.

Many studies have shown that HDL cholesterol has anti-inflammatory, antithrombotic, and antioxidant effects $(9,10)$. HDL cholesterol is classically known to have antiatherogenic effects. It performs this function, especially through lipid-loaded macrophages. In this way, the transport of cholesterol to the artery wall is prevented. It also inhibits the signaling of HDL cholesterol adhesion molecules. Thus, inflammatory cells, especially monocytes, are prevented from adhering to the vascular wall. It may also limit the inflammatory response through its direct effect on monocytes (9). Thanks to these mechanisms of action, HDL cholesterol has also been shown to be resistant to atrial myocyte cells $(10,24)$. At the time of

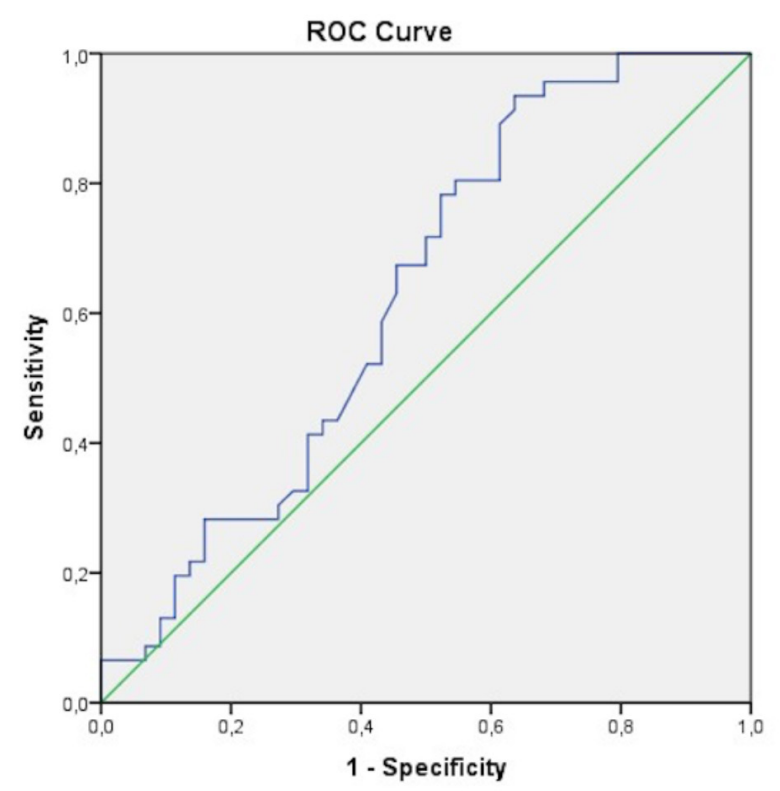

Diagonal segments are produced by ties.

Figure 2. Monocyte: The receiver operating characteristic (ROC) curve analysis for carotid artery stenosis ischemia, HDL cholesterol tries to limit inflammation with its anti-inflammatory and antioxidant activity. However, monocytes try to shift the process towards inflammation with their pro-inflammatory properties. Many studies have shown that inflammation can negatively affect the process of ischemia and vascular stenosis $(4,5,25)$. In our study, serum HDL cholesterol levels were lower in patients with carotid artery stenosis, supporting the anti-atherogenic effect of HDL cholesterol.

The monocyte/high-density lipoprotein ratio, a marker obtained by dividing the serum level of monocyte and HDL cholesterol, is an inflammatory indicator that was recently identified. Used more commonly for cardiovascular diseases initially, this marker was later shown to be associated with cerebrovascular disease and peripheral artery disease $(9,11,26)$. In a recent study, Bolayir et al. (11) showed that acute ischemic stroke patients with high MHR on hospitalization had higher mortality rates within 30 days. In the same study, the results of the ROC curve analysis demonstrated that the value of 17.5 was the cut-off value for 30-day mortality (11). Yurtdaş et al. (17) have shown that MHR levels were higher in stenosis than $50 \%$ in asymptomatic carotid artery stenosis. In this study, the MHR cut-off point was determined as 11.0 , with $75 \%$ sensitivity and $70 \%$ specificity based on $50 \%$ stenosis. In our study, the MHR level was significantly higher in patients with $50 \%$ and more symptomatic carotid stenosis. The MHR cutoff point was calculated as 15.22 with $66 \%$ sensitivity

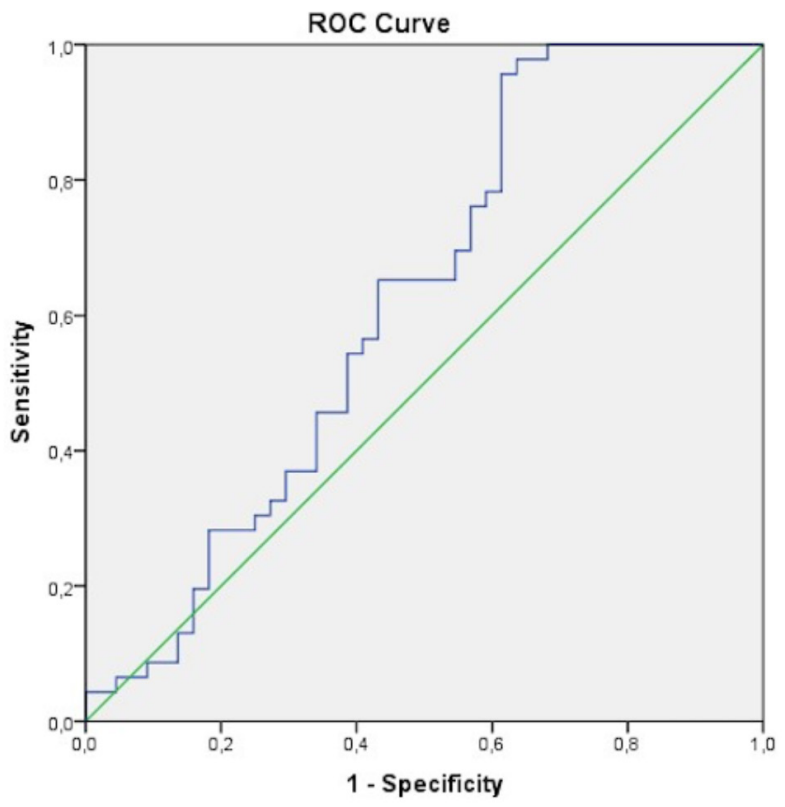

Figure 3. Monocyte/high-density lipoprotein (HDL) ratio (MHR): The receiver operating characteristic (ROC) curve analysis for carotid artery stenosis 
and 58\% specificity, demonstrating a relationship between the degree of stenosis and MHR level.

\section{Study Limitations}

This was a case-control and retrospective study with relatively few patients. The difference in the frequency of comorbid chronic diseases (such as hypertension and DM) between groups may affect the results. In addition, the relationship between MHR and symptom time has not been evaluated. Many factors affect monocyte and $\mathrm{HDL}$ cholesterol levels. Although diseases affecting these parameters were excluded, these parameters are also influenced by many local and/or systemic processes. Therefore, larger and multi-center studies are needed on this subject. Despite these limitations, to our knowledge, this is the first study to evaluate the MHR level in patients with symptomatic carotid artery stenosis. In addition, risk factors that may affect monocytes and HDL cholesterol levels were excluded from the study.

\section{Conclusion}

Previous studies have shown that monocyte and HDL cholesterol have direct and/or indirect effects on the vascular system, especially through inflammatory mechanisms. In our study, it was observed that HDL cholesterol and MHR levels were lower in patients with carotid artery stenosis. It was also demonstrated that monocyte and MHR levels were higher in patients with $50 \%$ or more symptomatic carotid stenosis. MHR is a predictive biomarker for the degree of symptomatic stenosis in the carotid artery.

\section{Ethics}

Ethics Committee Approval: Prior to the study, approval was obtained from the local ethics committee of Selcuk University (date: 21.04.2021, decision number: 2021/221).

Informed Consent: Since this study was retrospective, informed consent form did not required.

\section{Authorship Contributions}

Concept: F.E., A.T.Y., G.O., Gok.O., S.O., Design: F.E., A.T.Y., G.O., Data Collection and/or Processing: F.E., A.T.Y., G.O., Analysis and/or Interpretation: F.E., Gok.O., S.O., Literature Research: F.E., Gok.O., S.O., Writing: F.E., A.T.Y.

Conflict of Interest: No conflict of interest was declared by the authors.

Financial Disclosure: The authors declared that this study received no financial support.

\section{References}

1. Dharmakidari S, Bhattacharya P, Chaturvedi S. Carotid artery stenosis: Medical therapy, surgery, and stenting. Curr Neurol Neurosci Rep 2017;17:77.
2. Orrapin S, Rerkasem K. Carotid endarterectomy for symptomatic carotid stenosis. Cochrane Database Syst Rev 2017;6:CD001081.

3. Meschia JF, Klaas JP, Brown RD Jr, Brott TG. Evaluation and management of atherosclerotic carotid stenosis. Mayo Clin Proc 2017;92:1144-57.

4. Taleb S. Inflammation in atherosclerosis. Arch Cardiovasc Dis 2016;109:708-15.

5. Hansson GK, Libby P, Schönbeck U, Yan ZQ. Innate and adaptive immunity in the pathogenesis of atherosclerosis. Circ Res 2002;91:281-91.

6. Kazmierski R, Guzik P, Ambrosius W, Ciesielska A, Moskal J, Kozubski W. Predictive value of white blood cell count on admission for in-hospital mortality in acute stroke patients. Clin Neurol Neurosurg 2004;107:38-43.

7. Olivares R, Ducimetière P, Claude JR. Monocyte count: a risk factor for coronary heart disease? Am J Epidemiol 1993;137:49-53.

8. Murphy AJ, Westerterp M, Yvan-Charvet L, Tall AR. Antiatherogenic mechanisms of high density lipoprotein: effects on myeloid cells. Biochim Biophys Acta 2012;1821:513-21.

9. Yakar HI, Kanbay A. Could monocyte level/HDL cholesterol ratio predict cardiovascular diseases in patients with COPD? Niger J Clin Pract 2020;23:450-5.

10. Chen SA, Zhang MM, Zheng $M$, et al. The preablation monocyte/ high density lipoprotein ratio predicts the late recurrence of paroxysmal atrial fibrillation after radiofrequency ablation. BMC Cardiovasc Disord 2020;20:401.

11. Bolayir A, Gokce SF, Cigdem B, et al. Monocyte/high-density lipoprotein ratio predicts the mortality in ischemic stroke patients. Neurol Neurochir Pol 2018;52:150-5.

12. Dix JE, McNulty BJ, Kallmes DF. Frequency and significance of a small distal ICA in carotid artery stenosis. AJNR Am J Neuroradiol 1998;19:1215-8.

13. Wilterdink JL, Feldmann E. Carotid stenosis. A neurologist's perspective. Neuroimaging Clin N Am 1996;6:831-41.

14. Steinberg D, Witztum JL. Is the oxidative modification hypothesis relevant to human atherosclerosis? Do the antioxidant trials conducted to date refute the hypothesis? Circulation 2002;105:2107-11.

15. Moreno PR, Purushothaman KR, Fuster V, O'Connor WN. Intimomedial interface damage and adventitial inflammation is increased beneath disrupted atherosclerosis in the aorta: implications for plaque vulnerability. Circulation 2002;105:2504-11.

16. Ma Y, Mouton AJ, Lindsey ML. Cardiac macrophage biology in the steady-state heart, the aging heart, and following myocardial infarction. Transl Res 2018;191:15-28.

17. Yurtdaş M, Yaylali YT, Özdemir M. The role of monocyte to HDL ratio in predicting clinically significant carotid stenosis in patients with asymptomatic carotid artery disease. Rev Assoc Med Bras 2020;66:1043-8. 
18. Jin R, Yang G, Li G. Inflammatory mechanisms in ischemic stroke: role of inflammatory cells. J Leukoc Biol 2010;87:779-89.

19. Riksen NP, Netea MG. Immunometabolic control of trained immunity. Mol Aspects Med 2021;77:100897.

20. V M, Raveesha. Relation between Monocyte/High dansity lipoprotein(HDL) ratio in acute ischemic stroke severity. J Assoc Physicians India 2020;68:67.

21. Oh SW, Yi HJ, Lee DH, Sung JH. Prognostic significance of various inflammation-based scores in patients with mechanical thrombectomy for acute ischemic stroke. World Neurosurg 2020;141:710-7.

22. Fotakis $P$, Kothari $V$, Thomas $D G$, et al. Anti-inflammatory effects of HDL (High-Density Lipoprotein) in macrophages predominate over proinflammatory effects in atherosclerotic plaques. Arterioscler Thromb Vasc Biol 2019;39:253-72.
23. Schumski A, Ortega-Gómez A, Wichapong $K$, et al. Endotoxinemia accelerates atherosclerosis through electrostatic charge-mediated monocyte adhesion. Circulation 2021;143:254-66.

24. Bonacina F, Pirillo A, Catapano AL, Norata GD. HDL in immuneinflammatory responses: Implications beyond cardiovascular diseases. Cells 2021;10:1061.

25. Libby P. Inflammation in atherosclerosis-no longer a theory. Clin Chem 2021;67:131-42.

26. Ceyhun G, Engin MÇ. The monocyte/high density lipoprotein cholesterol ratio (MHR) as an indicator of the need for amputation in patients with peripheral artery disease developing critical limb ischemia. Angiology 2021;72:268-73. 